Wright State University

CORE Scholar

Physics Faculty Publications

Physics

8-1969

\title{
NMR Determination of the Conduction-Electron Hyperfine Interaction in Crystalline CdO
}

David C. Look

Wright State University - Main Campus, david.look@wright.edu

Follow this and additional works at: https://corescholar.libraries.wright.edu/physics

Part of the Physics Commons

\section{Repository Citation}

Look, D. C. (1969). NMR Determination of the Conduction-Electron Hyperfine Interaction in Crystalline CdO. Physical Review, 184 (3), 705-708.

https://corescholar.libraries.wright.edu/physics/752

This Article is brought to you for free and open access by the Physics at CORE Scholar. It has been accepted for inclusion in Physics Faculty Publications by an authorized administrator of CORE Scholar. For more information, please contact library-corescholar@wright.edu. 


\title{
NMR Determination of the Conduction-Electron Hyperfine Interaction in Crystalline $\mathrm{CdO}$
}

\author{
D. C. Look* \\ A erospace Research Laboratories, Wright-Patterson Air Force Base, Dayton, Ohio 45433
}

(Received 17 March 1969)

\begin{abstract}
Measurements of the $\mathrm{Cd}^{113}$ nuclear-spin-lattice relaxation time $T_{1}$ and Hall effect in crystalline CdO, a degenerate semiconductor, have yielded the contact hyperfine strength of the conduction electrons at the nuclei. The product $T_{1} T=168 \mathrm{sec}{ }^{\circ} \mathrm{K}$, independent of temperature $T$ and frequency $\nu$ for $T=1.4,4.2$, and $77-350^{\circ} \mathrm{K}$, and for $\nu=2-10 \mathrm{MHz}$. Taking the carrier concentration $N=2.6 \times 10^{19} \mathrm{~cm}^{-3}$ independent of temperature to within $3 \%$ at $4.2,77$, and $300^{\circ} \mathrm{K}$, and using an effective electron mass $m_{e}{ }^{*}=0.2 m_{e}$, we calculate an averaged electron probability density at the nucleus, $\left\langle\left|\phi_{F}(0)\right|^{2}\right\rangle=7 \times 10^{25} \mathrm{~cm}^{-3}$, normalized to unity in an atomic volume. A comparison with $\left\langle\left|\phi_{\mathrm{A}}(0)\right|^{2}\right\rangle$ in an isolated atom is interpreted to show that the Fermi level of the impurity band lies in the host-lattice conduction band. The Hall-effect data support this. The resonance frequency shift predicted from the Korringa relationship, $-0.017 \%$, is smaller than the observed shift, $-0.031 \%$. This is thought to be due to covalency contributions rather than to electron-electron interactions.
\end{abstract}

\section{INTRODUCTION}

$A^{1}$ LTHOUGH CdO is generally considered to be a wide-band-gap (2.4-eV) semiconductor, it has a high conductivity $\left(10^{3} \Omega^{-1} \mathrm{~cm}^{-1}\right)$ due to donor impurity centers. ${ }^{1-3}$ These centers are probably $\mathrm{Cd}$ interstitials (or, possibly, $\mathrm{O}$ vacancies) arising from the stable solid solutions of $\mathrm{CdO}$ and $\mathrm{Cd}$. Above a certain concentration of excess $\mathrm{Cd}$, the donor wave functions overlap and form an "impurity band" (i.e., continuum of energy states) in which the electrons are degenerate. This critical concentration $N_{c}$ was given by $\operatorname{Mott}^{4}$ as $N_{c} \simeq\left(0.25 / a_{H}\right)^{3}$, where $a_{H}$ is the impurity Bohr radius. Above another concentration, $N_{c \mathrm{~b}}$, the Fermi level of the impurity band passes into the host-lattice conduction band. ${ }^{5}$ It is well $\mathrm{known}^{2,3}$ that the conduction electrons in $\mathrm{CdO}$ are degenerate, but it is not known whether $N$ is greater or less than $N_{\mathrm{cb}}$.

Nuclear magnetic resonance has been used to study such effects in doped samples of Si and Ge by investigating ${ }^{6,7}$ the Knight shifts (NMR frequency shifts due to conduction electrons), spin-lattice relaxation times, and EPR lines as a function of impurity concentration; for $N<N_{\mathrm{cb}}$, the Knight shift decreases rapidly in these samples, showing that the carrier wave functions no longer interact strongly with nuclei in the bulk, far from the impurity centers. In $\mathrm{CdO}$, however, the impurity concentration in single crystals is not easily

\footnotetext{
* Present address: University of Dayton, Dayton, Ohio mailing address: Aerospace Research Laboratories (ARX),

Wright-Patterson Air Force Base, Dayton, Ohio 45433.

1 E. Mollwo and R. Stump, Z. Physik 184, 286 (1965).

${ }^{2}$ R. W. Wright, J. Phys. B66, 273 (1953).

${ }^{3}$ For a comprehensive summary of data and a list of references on $\mathrm{CdO}$, see $\mathrm{M}$. Neuberger, Electronic Properties Information Center Report No. DS-149, 1966 (unpublished). Copies may be obtained from Defense Documentation Center (DDC), Cameron Station, BIdg. 5, 5010 Duke St., Alexandria, Va. 22314.

${ }^{4}$ N. F. Mott, Can. J. Phys. 34, 1356 (1956).

5 E. M. Conwell, Phys. Rev. 103, 51 (1956).

${ }^{6}$ R. K. Sundfors and D. F. Holcomb, Phys. Rev. 136, A810 (1964).

${ }^{7}$ M. N. Alexander and D. F. Holcomb, Rev. Mod. Phys. 40, 815 (1968). Further references may be found in this article.
}

controlled, and, furthermore, there appears to be an additional paramagnetic-resonance-frequency shift due to covalency effects, which obscures the Knight shift. Therefore, the author has approached the problem through a study of the nuclear-spin-lattice relaxation time $T_{1}$ and Hall effect as a function of temperature. From these data we can calculate a value for $\left\langle\left|\phi_{F}(0)\right|{ }^{2}\right\rangle$, the averaged conduction-electron probability density at $\mathrm{Cd}^{113}$ nuclei in the bulk, and infer that it is characteristic of the host-lattice conduction band.

\section{NMR RELAXATION AND KNIGHT SHIFT}

\section{A. Theory}

The contact term of the conduction-electron hyperfine interaction is often an effective mechanism for nuclear-spin-lattice relaxation. The Hamiltonian may be written ${ }^{8}$

$$
\mathfrak{F}_{1}=\frac{8 \pi}{3} \gamma_{e} \gamma_{n} \hbar^{2} \sum_{j, l} \mathbf{I}_{j} \cdot \mathbf{S}_{l} \delta\left(\mathbf{r}_{j l}\right)
$$

where $\gamma_{e}$ and $\gamma_{n}$ are, respectively, the electron and nuclear gyromagnetic ratios, $h$ is Planck's constant divided by $2 \pi$, and $\mathbf{I}_{j}, \mathbf{S}_{l}$, and $\mathbf{r}_{j l}$ are, respectively, the $j$ th nuclear spin, the $l$ th electron spin, and the vector between them. Using the nuclear Zeeman energy as a zero-order Hamiltonian, and applying standard timedependent perturbation theory, we can calculate ${ }^{8}$ the nuclear-spin-lattice relaxation time $T_{1}$. For nondegenerate electrons it may be shown that $T_{1} \propto N^{-1} T^{-1 / 2}$, where $N$ is the carrier concentration and $T$ is the absolute temperature; for degenerate electrons, $T_{1} \propto N^{-2 / 3} T^{-1}$. Thus measurements of $T_{1}$ as functions of $N$ and $T$ are useful in determining the degree of degeneracy of the electrons. Specifically, for degenerate electrons and $k T \ll E_{F}$,

$$
1 / T_{1}=\left(64 \pi^{3} / 9\right) \gamma_{e}^{2} \gamma_{n}^{2} h^{3}\left\langle\left|\phi_{F}(0)\right|^{2}\right\rangle^{2} \rho^{2}\left(E_{F}\right) k T,
$$

\footnotetext{
${ }^{8}$ See, for example, A. Abragam, The Principles of Nuclear Mag.
} netism (Oxford University Press, London, 1961), p. $355 \mathrm{ff}$. 
where $k$ is Boltzmann's constant, $\rho\left(E_{F}\right)$ is the electronic density of states at the Fermi level (for one spin direction), and $\left\langle\left|\phi_{F}(0)\right|^{2}\right\rangle$ is the electronic probability density at the nucleus, averaged over all states on the Fermi surface. Assuming the independent-electron band model, ${ }^{9}$

$$
\rho\left(E_{F}\right)=\frac{3 N V}{4} \frac{3}{E_{F}}=\left(\frac{3}{8 \pi^{4}}\right)^{1 / 3} \frac{m_{e}^{*}}{\hbar^{2}} N^{1 / 3} V,
$$

where $V$ is the sample volume and $m_{e}{ }^{*}$ is the band effective mass. Inserting Eq. (3) into Eq. (2), and using the measured ${ }^{10} \gamma_{e}=1.57 \times 10^{7}$ and $\gamma_{n}=5.93 \times 10^{3}$ (for $\left.\mathrm{Cd}^{113}\right)$, we get

$$
1 / T_{1}=5.9 \times 10^{33}\left(m_{e}^{*}\right)^{2} V^{2}\left\langle\left|\phi_{F}(0)\right|^{2}\right\rangle^{2} N^{2 / 3} T .
$$

The polarization of the Fermi-level electrons in the applied magnetic field gives rise to a resonance field shift, the Knight shift, ${ }^{11}$ given for independent electrons by

$$
\Delta H / H \equiv K=-\frac{4}{3} \pi \gamma_{e}^{2} \hbar^{2}\left\langle\left|\phi_{F}(0)\right|^{2}\right\rangle_{\rho}\left(E_{F}\right) .
$$

From Eqs. (2) and (5) we get the Korringa product, for independent electrons,

$$
T_{1} T K^{2}=\frac{\gamma_{e}^{2}}{\gamma_{n}^{2}} \frac{\hbar}{4 \pi k}=5.36 \times 10^{-6} \text { for } \mathrm{CdO} .
$$

If our resonance field shift is really due to conduction electrons, it should satisfy Eq. (6) if electron-electron interactions can be neglected.

\section{B. Experimental Considerations and Results}

The CdO crystals were grown by the vapor-phase deposition method with a vaporization temperature of about $1200^{\circ} \mathrm{C}$ and a condensation temperature of about $1000^{\circ} \mathrm{C}$. The modification is always face-centered cubic (halite). The crystals typically appeared as thin $(0.5 \mathrm{~mm})$, single-crystal plates, often joined at right angles, forming a "square" spiral. The largest singlecrystal plate (on which Hall measurements were done) was about $9 \times 3.5 \times 0.6 \mathrm{~mm}$. For the NMR measurements, the crystals were crushed to about $1-\mathrm{mm}^{3}$-size particles and then added together to make a sample of about $1-\mathrm{cm}^{3}$ volume.

The basic NMR spectrometer was a Varian V-4200B wide-line system equipped with a variable-temperature accessory for operation from 85 to $550^{\circ} \mathrm{K}$. Liquidhelium experiments were performed in a specially constructed Pyrex Dewar to which the NMR probe was clamped. The higher temperatures were measured by means of a thermocouple inserted into the sample.

The resonance field shift of the $\mathrm{Cd}^{113}$ nuclei was measured with respect to a solution of $\mathrm{CdCl}_{2}$. It was

\footnotetext{
${ }^{9}$ See, for example, C. Kittel, Introduction to Solid State Physics (John Wiley \& Sons, Inc., New York, 1953), pp. 249 and 250.

${ }_{10}$ B. Elschner and M. Schlaak, Phys. Letters 24A, 10 (1967).

${ }^{11}$ See Ref. 8, p. 199.
}

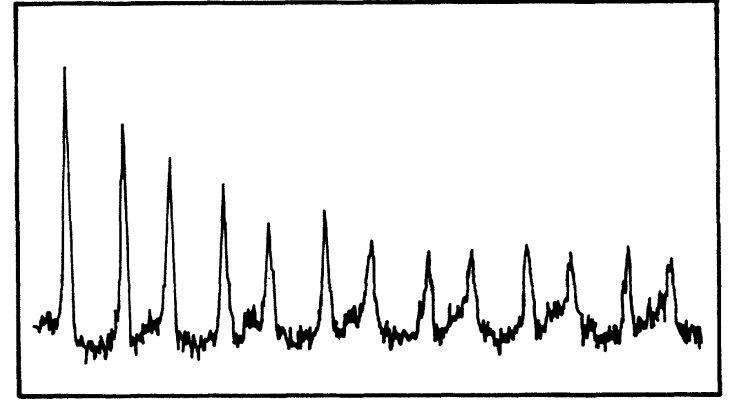

FIG. 1. Recorder tracing of a typical sequence of $\mathrm{Cd}^{113}$ absorption curves in $\mathrm{CdO}$ produced by $6 \frac{1}{2}$ cycles of 30 -cps field modulation (13 passes through the resonance field) after being off resonance for a time $4 T_{1}$. In this case, 150 sequences have been added in a time-averaging computer to enhance the signal-tonoise ratio. (See Ref. 13 for the details of calculating $T_{1}$ from such a sequence.)

$\Delta H / H=(-0.031 \pm 0.003) \%$, independent of field (2-10 $\mathrm{kG})$ and temperature $\left(4.2-300^{\circ} \mathrm{K}\right)$, and consistent with the results of Anderson and Corll. ${ }^{12}$ The $T_{1}$ measurements were carried out by the field-tone-burst method ${ }^{13}$ using a time-averaging computer for signal enhancement. The tone-burst system consisted of a Wavetek model 111 waveform generator, a General Radio model 1396B tone-burst generator, and a Hewlett-Packard model 6824A programmable, current-regulated power supply to drive the probe's modulation coils. A typical sequence of absorption curves, from which $T_{1}$ is calculated, ${ }^{13}$ appears in Fig. 1.

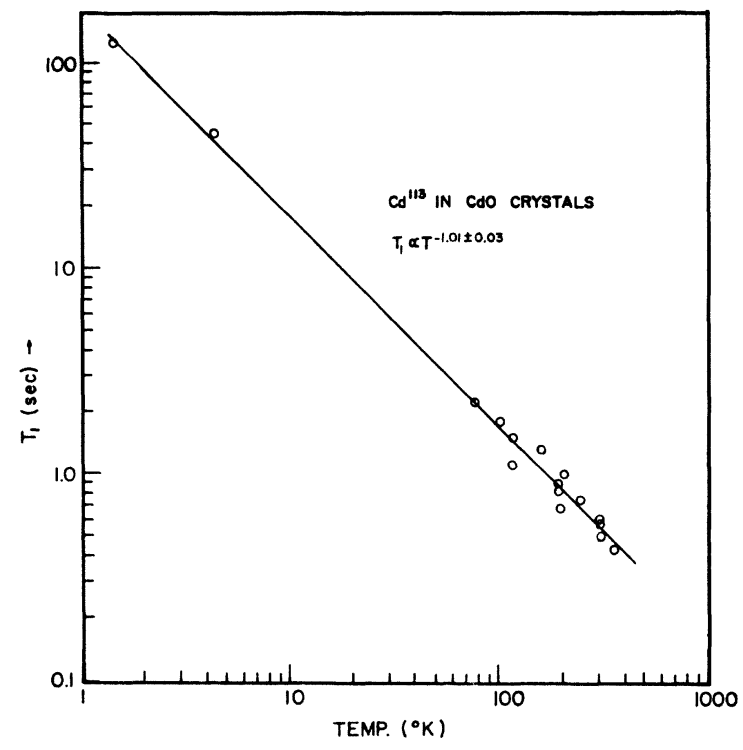

FIg. 2. Nuclear-spin-lattice relaxation time as a function of temperature in a sample of $\mathrm{CdO}$ crystals. It is seen that $T_{1} \propto T^{-1}$.

12 D. H. Anderson and J. A. Corll, Bull. Amer. Phys. Soc. 11, 482 (1966).

${ }^{13}$ D. C. Look and D. R. Locker, Phys. Rev, Letters 20, 987 (1968). 
The $T_{1}$-versus- $T$ data are presented in Fig. 2. The result is $T_{1} T=168 \pm 10 \mathrm{sec}^{\circ} \mathrm{K}$, independent of temperature $\left(1.4-350^{\circ} \mathrm{K}\right)$ and frequency $(2-10 \mathrm{MHz})$.

\section{HALL-EFFECT MEASUREMENTS}

The electrical measurements were carried out at 4.2 , 77 , and $300^{\circ} \mathrm{K}$ on a single crystal, $9 \times 3.5 \times 0.6 \mathrm{~mm}$. The five-contact method ${ }^{14}$ was used and indium leads were soldered on with an ultrasonic iron. Current-voltage characteristics indicated Ohmic conditions at all temperatures. The Hall-effect apparatus was of standard design and was used with a magnetic field of $11 \mathrm{kG}$ and a crystal current of $200 \mathrm{~mA}$. These parameters gave crystal voltages of about $10 \mathrm{mV}$ and Hall voltages of about $0.1 \mathrm{mV}$. The Hall voltages reversed (to within $2 \%$ ) with magnetic field reversal at 300 and $77^{\circ} \mathrm{K}$, but at $4.2^{\circ} \mathrm{K}$ the absolute magnitude of the two voltages differed by about $25 \%$. However, their signs were reversed. An average of the two absolute magnitudes was taken at each temperature to compensate for the magnetoresistive effects.

The results are shown in Table I. The Hall constant for degenerate electrons is $R=1 / \mathrm{Nec}$. It is seen that from 300 to $4.2^{\circ} \mathrm{K}$ the resistivity and mobility increase and decrease, respectively, by about $10 \%$, while the carrier concentration changes by only $3 \%$ (within the limits of precision). Of course, it should be noted that while the precision error is only about $5 \%$, the accuracy error is much larger (perhaps 15\%) due to geometrical considerations, e.g., the finite area of the contacts and variations in the crystalline cross section.

Miloslavskii and Ranyuk ${ }^{15}$ have used infrared absorption to investigate the mobility of thin layers of $\mathrm{CdO}$, produced by cathode sputtering. They found that their results could be explained by assuming that the mobility is due to scattering of degenerate electrons by impurity ions, according to the Conwell-Weisskopf relation

$$
\mu=\frac{4 e a_{i}}{\pi p_{F}} \frac{\left(2 E_{F} / E_{i}\right)^{2}}{2 \ln \left[1+\left(2 E_{F} / E_{i}\right)^{2}\right]} .
$$

Here $p_{F}=\pi \hbar(3 N / \pi)^{1 / 3}, \quad E_{F}=\left(\pi^{2} \hbar^{2} / 2 m_{e}{ }^{*}\right)(3 N / \pi)^{2 / 3}$ $a_{i}=N_{i}^{-1 / 3}$, and $E_{i}=2 e^{2} / \kappa a_{i}$, where $\kappa$ is the dielectric constant and $N_{i}$ is the impurity concentration. Assuming ${ }^{3}$ $\kappa \simeq 6$ and $N_{i}=N$, i.e., a single electron associated with each impurity ion, we can calculate for our sample $m_{e}{ }^{*} \simeq 0.17 m_{e}$ to $0.19 m_{e}$, covering the narrow range of our measured mobilities. In fact, Miloslavskii and Ranyuk determined their effective mass at the bottom of the conduction band to be $m_{e}{ }^{*} \simeq 0.2 m_{e}$, in good agreement with ours.

Values of $m_{e}{ }^{*}$ ranging from $0.1 m_{e}$ to $0.3 m_{e}$ have been given by Wright and Bastin ${ }^{16}$ using other types of mea-

\footnotetext{
${ }^{14}$ See, for example, E. H. Putley, The Hall Effect (Butterworths Scientific Publications Ltd., London, 1960), p. $42 \mathrm{ff}$.

${ }^{15}$ V. K. Miloslavskii and A. I. Ranyuk, Opt. i Spektroskopiya
}

TABLE I Resistivity $\rho$, mobility $\mu$, and carrier concentration $N$ in a single crystal of $\mathrm{CdO}$.

\begin{tabular}{cccc}
\hline $\begin{array}{c}\text { Temp. } \\
\left({ }^{\circ} \mathrm{K}\right)\end{array}$ & $\begin{array}{c}\rho \\
(\Omega \mathrm{cm})\end{array}$ & $\begin{array}{c}\mu \\
\left(\mathrm{cm}^{2} / \mathrm{V} \mathrm{sec}\right)\end{array}$ & $\begin{array}{c}N \\
\left(\mathrm{~cm}^{-3}\right)\end{array}$ \\
\hline 300 & $1.98 \times 10^{-3}$ & 122 & $2.58 \times 10^{19}$ \\
77 & $2.04 \times 10^{-3}$ & 117 & $2.62 \times 10^{19}$ \\
4.2 & $2.17 \times 10^{-3}$ & 108 & $2.66 \times 10^{19}$ \\
\hline
\end{tabular}

surements, for example, Hall effect $\left(0.3 m_{e}\right)$, resistivity $\left(0.1 m_{e}\right)$, thermoelectric power $\left(0.1 m_{e}\right.$ to $\left.0.2 m_{e}\right)$, and transverse magnetoresistance $\left(0.1 m_{e}\right)$. Each of these values is deduced, however, from theory which takes into account only optical-mode scattering of the electrons, which is clearly not the dominant mechanism in our sample. Thus, in view of the relative simplicity of interpretation of the optical method and the agreement of their results with ours, according to Eq. (7), we conclude that our effective mass is given by $m_{e}^{*}=0.2 m_{e}$, probably to within $20 \%$.

\section{DISCUSSION}

Using $m_{e}^{*} \simeq 0.2 m_{e}$ and $N \simeq 2.6 \times 10^{19} \mathrm{~cm}^{-3}$, we calculate from Eq. (4), $V\left\langle\left|\phi_{F}(0)\right|^{2}\right\rangle \simeq 1800$, the wave function being normalized to unity in a unit volume. This number is the relative electronic probability density at a nucleus compared to the average value in the crystal. If we normalize to unity in an atomic volume, then $\left\langle\left|\phi_{F}(0)\right|^{2}\right\rangle \equiv P_{F} \simeq 7 \times 10^{25} \mathrm{~cm}^{-3}$. This value could be improved by a better determination of the effective mass but the conclusions drawn in this paper would not be affected.

We compare $P_{F}$ with $P_{A} \equiv\left\langle\left|\phi_{A}(0)\right|^{2}\right\rangle$, the value in the free ion. $P_{A}$ may be calculated using the Goudsmit formula ${ }^{17}$ and, if $\mathrm{CdO}$ is mostly ionic, the $4 d^{10} 5 s^{2} S_{1 / 2}$ term is probably the most appropriate one; this gives $P_{A} \simeq 9.2 \times 10^{25} \mathrm{~cm}^{-3}$. The factor $\xi \equiv P_{F} / P_{A} \equiv 0.8$ is in the range typical for metals. ${ }^{8}$ If, indeed, the electrons were localized around the impurity centers, we would expect a much lower $\xi$, since there would be little interaction with nuclei in the bulk. Thus, the electrons appear to be more characteristic of the conduction band than of an isolated impurity band. Furthermore, the Hall-effect data support this, since a "hump" is expected in $R(=1 / N e c)$ versus $T$ if the impurity band does not overlap the conduction band. ${ }^{5,18}$ Although detailed $N$-versus- $T$ measurements were taken only at the temperatures mentioned in Sec. III, the Hall voltage was observed continuously and appeared constant as the sample warmed from 4.2 to $300^{\circ} \mathrm{K}$.

It has been shown, therefore, that the electrons in our $\mathrm{CdO}$ sample are degenerate for $1.4<T<350^{\circ} \mathrm{K}$, since

11, 536 (1961) [English transl.: Opt. Spectry. (USSR) 11, 289 (1961)].

${ }^{16}$ R. W. Wright and J. A. Bastin, Proc. Phys. Soc. (London) 71, 109 (1958).

${ }^{17}$ S. Goudsmit, Phys. Rev. 43, 636 (1933).

${ }^{18}$ N F. Mott. and W. D. Twose, Advan. Phys. 10, 107 (1961). 
$T_{1} T$ is constant. ${ }^{8}$ Moreover, the magnitude of the ratio $P_{F} / P_{A}$ and the lack of a maximum in the $R$-versus- $T$ data are interpreted to show that the Fermi level of the impurity band due to the excess Cd has crossed over into the conduction band of the host $\mathrm{CdO}$ lattice, i.e.,

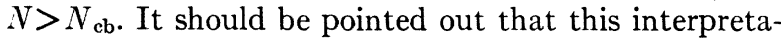
tion is not conclusive, since it is not certain that all materials will exhibit the same changes in $P_{F}$ (as $N$ passes through $V_{\mathrm{cb}}$ ) that have been observed ${ }^{6} 7$ in $\mathrm{Si}: \mathrm{P}$ and other substances and that we have assumed will occur in CdO. Also, the lack of a Hall-effect "hump" has not been shown to be a universal phenomenon occurring only in substances with $N>N_{\mathrm{cb}}$. Nevertheless, we believe our interpretation is quite plausible and is, in fact, correct. It should also be noted that it is necessary that the condition $N>N_{\mathrm{cb}}$ be consistent with the weaker condition $N>N_{c}$, the Mott criterion. Using $m_{e}{ }^{*} \simeq 0.2 m_{e}$ and the dielectric constant ${ }^{3} \kappa \simeq 6$, we can calculate $a_{H}=\kappa h^{2} / m_{e}{ }^{*} e^{2} \simeq 16 \AA$, giving $N_{c} \simeq\left(0.25 / a_{H}\right)^{3}$ $\simeq 4 \times 10^{18} \mathrm{~cm}^{-3}$; thus, $N>N_{c}$, as required.

If, as we have asserted, $P_{F}\left(\mathrm{Cd}^{113}\right)$ is characteristic of the $\mathrm{CdO}$ conduction band, it should be possible to compare our experimental value with theoretical band calculations. Maschke and Rössler have recently performed such a calculation, ${ }^{19}$ although they did not compute $P_{F}{ }^{20}$

The theoretical Korringa product [Eq. (6)] and our $T_{1}$ data predict a line shift due to the conduction electrons of $\Delta H / H \simeq-0.017 \%$, while the measured value is $-0.031 \%$. Such a discrepancy is quite commonly observed in metals where electron-electron interactions are important. ${ }^{21}$ However, these effects are probably not large in our sample with only $10^{19}$ electrons per $\mathrm{cm}^{3}$. The extra paramagnetic shift is probably a chemical

\footnotetext{
${ }^{19} \mathrm{~K}$. Maschke and U. Rössler, Phys. Status Solidi 28, 577 (1968)

${ }^{20}$ K. Maschke and U. Rössler (private communication)

${ }^{21}$ D. Pines, in Solid State Physics, edited by F. Seitz and

D. Turnbull (Academic Press Inc., New York, 1956), Vol. 1.
}

shift ${ }^{22}$ caused by the $p$-type electrons which contribute to the covalency ( $\mathrm{CdO}$ is believed to have about $20 \%$ covalent bonding ${ }^{23}$. This explanation is reasonable when the $\mathrm{CdO}$ shift is compared with that measured (see Ref. 12) in some samples of her sister compounds CdS, $\mathrm{CdSe}$, and CdTe. These samples have paramagnetic shifts ranging from -0.013 to $-0.051 \%$, but carrier concentrations of $10^{6}-10^{15} \mathrm{~cm}^{-3}$, not nearly enough to account for a measureable part of their shifts. Thus, it is likely that $\mathrm{CdO}$ also should have an appreciable paramagnetic contribution other than that from its conduction electrons. It is unlikely that there is much contribution to either $T_{1}$ or $\Delta H$ from other mobile electrons, since the conduction band in $\mathrm{CdO}$ should have little $p$ character, and also since orbital and core-polarization terms are usually quite small in nontransition metals. ${ }^{24}$

Future programs to further elucidate the properties of $\mathrm{CdO}$ include a better determination of the effective mass, which is not very important until band theorists give an accurate value for $P_{F}\left(\mathrm{Cd}^{113}\right)$, and growth of crystals with lower impurity concentrations. It may be possible to grow a series of crystals with $N$ ranging from less than $N_{c}$ to greater than $N_{\mathrm{cb}}$, and these parameters can thus be investigated by studies of $T_{1}$ and $\Delta H / H$ versus $N$.

\section{ACKNOWLEDGMENTS}

The author would especially like to thank Donald Locker for many helpful discussions regarding experimental aspects of the work and the nature of $\mathrm{CdO}$. Gratitude is also expressed to Don Zimmerman for performing the frequency-shift measurements and to Don Naas for growing the crystals used in this study.

${ }^{22}$ See, for example, C. J. Jameson and H. S. Gutowsky, J. Chem. Phys. 40, 1714 (1964).

${ }^{23} \mathrm{~J}$. C. Phillips and J. A. Van Vechten, Phys. Rev. Letters 22, 705 (1969)

${ }_{24}$ R. J. Noer and W. D. Knight, Rev. Mod. Phys. 36, 177 (1964). 